\title{
Immunohistochemical Markers in Benign Odontogenic Tumors
}

\author{
${ }^{1}$ Shraddha S Walekar, ${ }^{2}$ Shrikant R Sonune, ${ }^{3}$ Janaki S Iyer, ${ }^{4}$ Rashmi Hosalkar, ${ }^{5}$ Yogita Murudkar
}

\begin{abstract}
Odontogenic tumours includes broad spectrum of lesions derived from specialized dental tissues. Neoplasms and tumours related to the odontogenic apparatus may be composed only of epithelial tissue or epithelial tissue associated with odontogenic ectomesenchyme. They constitute abortive attempt at odontogenesis. Odontogenic tumors can be intraosseous or extraosseous with varying clinical and radiographic features. Odontogenic tumours are associated with molecular and genetic alterations. This review presents outline of molecular and genetic alterations associated with nature and growth of benign odontogenic tumours.
\end{abstract}

Key words: Ameloblastoma, Amelogenin, Cytokeratin, Immunohistochemical markers, Odontogenic tumors

How to cite this article: Walekar SS, Sonune SR, lyer JS, Hosalkar R, Murudkar Y. Immunohistochemical Markers in Benign Odontogenic Tumors. J Contemp Dent 2018;8(2):101105.

\section{Source of support: Nil}

Conflict of interest: None

\section{INTRODUCTION}

Pathologies of the tooth forming apparatus comprise of epithelial, ectomesenchymal, and/or mesenchymal elements, that give rise to odontogenic tumors. Odontogenic tumors can be

Ddental hamartomas, benign or malignant tumors. These tumors, therefore, are found exclusively within jaw bones or in the soft mucosal tissue overlying the tooth-bearing areas. The progress in the field of molecular pathology has brought the remarkable change in the practice of pathology. Many studies have been done from biochemistry to molecular biology, and this gave rise to various tumor markers.

\footnotetext{
${ }^{1,3,4}$ Lecturer, ${ }^{2}$ Dental Surgeon, ${ }^{5}$ Tutor

1,3-5 Department of Oral Pathology and Microbiology, MGM Dental College and Hospital, Navi Mumbai, Maharashtra, India

${ }^{2}$ Department of Oral Pathology, Sub-District Hospital, Solapur, Maharashtra, India

Corresponding Author: Shraddha S Walekar, Lecturer, Department of Oral Pathology and Microbiology, MGM Dental College and Hospital, Navi Mumbai, Maharashtra, India, e-mail: shraddhawalekar7@gmail.com
}

According to the National Cancer Institute (2008), "Tumor markers are substances produced by tumor cells or by other cells of the body in response to cancer or certain benign (non-cancerous) conditions. These substances can be found in the blood, in the urine, in the tumor tissue, or in othertissues." ${ }^{1}$ Different tumor markers are found in different cancers and levels of the same tumor marker can be altered in more than one type of cancer. CD10, matrix metalloproteinases, cytokeratins, vimentins are few tumor markers. ${ }^{2}$

Modern pathology has integrated tumor markers. So this article reviews regarding various tumor markers in benign odontogenic lesions.

\section{Amelogenin}

Enamel matrix includes significant protein components: Amelogenins and non-amelogenins (enamelins, ameloblastins, and tuftelins) $)^{3}$. These are produced by ameloblast. Amongst enamel proteins, amelogenin are major proteins. They contain $90 \%$ extracellular matrix and play a role in the organization of enamel. The cytoplasm of the cells of the reduced enamel epithelium, stratum intermedium and stellate reticulum of the enamel organ demonstrated amelogenin. ${ }^{4}$ Odontogenic tumors contain odontogenic epithelium. Studies suggest that aberrated enamel proteins are seen in oncogenesis. Anigol et al. studied amelogenin expression in odontogenic cysts and tumors. According to them, amelogenin expression was positive in the dentigerous cyst, radicular cyst, odontogenic keratocyst (OKC), ameloblastoma, squamous odontogenic tumor, adenomatoid odontogenic tumor (AOT), calcifying epithelial odontogenic tumor (CEOT), odontomas and ameloblastic carcinoma. ${ }^{5}$ They concluded that amelogenin molecule could be used as a marker for odontogenic lesions and odontogenic epithelium especially when there is difficulty in a demonstration of the same in routine hematoxylin and eosin stains. ${ }^{5}$ Calcified globular structures and adjacent lesional cells showed an intensely positive reaction. No other hard tissue like bone showed expression of amelogenin. Amelogenin expression in hard tissue form ative lesions may indicate the advancing differentiation of the tissue. ${ }^{5}$

\section{Cytokeratins}

Intermediate filaments of epithelial cells are known as cytokeratin and of mesenchymal cells is vimentin. Twenty 
different types of cytokeratins are seen in human beings. Different epithelia have different types of cytokeratins. Simple epithelia and occasionally basal and suprabasal cells of stratified epithelium consist of cytokeratin 7 and 9. Cytokeratin 8 and 18 is seen in embryonic tissue and simple epithelia. Suprabasal layers of stratified squamous epithelium contain cytokeratin 10 and 13 and basal layer consist of cytokeratin 14. According to Gao et al., CK 5, 7, 8, 13, 14, 17 and 19 were present in the human enamel organ. Gao et al. studied immunohistochemical expression of CK 7, 8, 10, 13, 14,18 , and 19 in tooth germs, dental follicles with reduced enamel epithelium, ameloblastomas, AOTs, CEOTs, ameloblastic fibromas (AF), and odontomas. They showed that CK14 was the main intermediate filament in the odontogenic epithelium of dental lamina, reduced enamel epithelium, almost all cells of enamel organ. Stellate reticulum like cells and areas of squamous metaplasia expressed CK13. Variable expression was observed with CK $7^{6,7}$

\section{Tenascin}

Extracellular matrix glycoprotein regulating cell morphology is tenascin.

It is commonly expressed during epithelial-mesenchymal interaction during tooth development. Similarly, the stromal expression is seen in carcinomas and epithelialmesenchymal interaction during carcinogenesis. ${ }^{8}$ Previous studies suggest that tenascin was localised in human teeth during epithelial bud formation. It was seen in the basement membrane during odontoblast differentiation. Tenascin was not seen in dentine as dental papilla cells stop expressing on differentiating into dentin. The unusual expression of tenascin in dental mesenchymal cells shows its capacity to differentiate into hard tissue forming cells. ${ }^{9,10}$ Nagai et al. studied the expression of tenascin in ameloblastomas, ameloblastic fibromas, ameloblastic carcinomas and tooth germs using monoclonal antibodies. ${ }^{8}$ Tenascin expression was observed in the dental papilla under the basement membrane, pre-odontoblastic layer and negative in a dental follicle. In ameloblastic fibroma, the basement membrane and stroma showed high tenascin expression. In follicular ameloblastoma, irregular linear tenascin positive reaction was observed in the basement membrane zone. In ameloblastic carcinomas, stroma and basement membrane showed an irregular strong reaction. They concluded that tenascin can be used as a marker in the epithelialmesenchymal interaction during tooth development and in odontogenic tumors. ${ }^{11}$

\section{Bone Morphogenetic Proteins}

Cytokine transforming growth factor beta superfamily includes bone morphogenetic protein. Bone morpho- genetic protein is observed in bone and dentin matrix. It has the ability to induce bone formation. ${ }^{12}$ Previous literature suggests that bmp 2, 4, 7 can be expressed within the enamel knot which acts as signaling center. ${ }^{13}$ Gao et al. studied the expression of bone morphogenetic proteins in 44 cases of odontogenic tumors consisting of 20 ameloblastomas, eight cementifying fibromas, five benign cementoblastomas, three dentinomas and two cases of compound odontomas, adenomatoid odontogenic tumors, calcifying epithelial odontogenic, and odontogenic fibromas. They classified odontogenic tumors according to the expression observed. Positive expression was observed in all cementifying fibromas, benign cementoblastomas, dentinomas, odontogenic fibromas, and compound odontomas whereas all ameloblastomas, adenomatoid odontogenic tumors, and calcifying epithelial odontogenic tumors were negative. They concluded that BMPMcAb-positive odontogenic tumors were those tumors with the formation of hard tissues. ${ }^{14}$

\section{Receptor Activator of Nuclear Factor-KappaB Ligand (RANKL)/RANK/Osteoprotegerin (OPG)}

Although most odontogenic tumors are benign, they are locally destructive and cause significant bone destruction. Members of tumor necrosis factor superfamily cause bone remodeling. These include RANK-RANKL and osteoprotegerin (OPG). They control activation, formation, and differentiation of osteoclasts. ${ }^{15}$ Receptor activator of NF-KB ligand is expressed by osteoblast and stromal cells. RANKL binds to its receptor RANK present on the surface of osteoclasts and precursors and differentiates osteoclasts into multinucleated cells to bring bone resorption. Osteoblast and stromal cells also secrete osteoprotegerin which binds Ra nkl and prevents bone resorption. ${ }^{16}$ Andrade et al. studied the expression of RANK, RANKL and OPG expression in calcifying cystic odontogenic tumor (CCOT), AOT, CEOT, odontogenic myxoma (OM), and ameloblastic fibroma (AF). They observed higher expression of OPG in AOT and CCOT. On the contrary, mesenchymal cells of OM and AF expressed RANKL which was consistent with their tendency for recurrence and bone resorption. ${ }^{17}$

\section{Matrix Metalloproteinase}

The group of enzymes that bring about the degradation of extracellular matrix proteins during growth and tissue remodeling is Matrix Metalloproteinase (MMP) ${ }^{18}$ The classification of these enzymes is based on the type of substrates like collagenase, gelatinase, stromelysin. MMP-2, also known as gelatinase, has substrates gelatin Types I to III and collagen types IV, V, VII, and X. MMP-3, also known as stromelysin degrades proteoglycans, 
fibronectin, laminin.Bast et al. studied the expression of MMP in OM's. They observed significant expression of MMP-2 in tumor cells.They concluded that MMP-2 aids in expansion and growth of a tumor. ${ }^{19}$ Pinheiro et al. studied the expression of MMP in ameloblastoma using immunohistochemistry and biochemical methods. They confirmed the expression of MMP-1, MMP-2, and MMP9 in ameloblastomas. According to Pinheiro et al., MMP expression was seen in peripheral columnar cells, bone neoplasm interface, in stromal regions and bone. The secretion of MMP is either through ameloblastoma cells or induction by stromal cells. MMP's releases mitogens from bone by causing its resorption and matrix solubilization. These mitogens further increase cellular proliferation rate of ameloblastoma in a cord like fashion. ${ }^{20}$

\section{Collagen IV $\alpha 1$ to $\alpha \mathbf{6}$ Chains}

Type IV collagen is a major component of the basement membrane. It is a triple helical molecule consisting of three $\alpha$ - chains. They are divided as $\alpha 1$-like class composed of $\alpha 1$ (IV), $\alpha 3$ (IV) and $\alpha 5$ (IV) chains; and $\alpha 2$-like class composed of $\alpha 2$ (IV), $\alpha 4$ (IV) and $\alpha 6$ (IV). It is encoded by six distinct genes, COL4A1 to COL4A6. During tooth development, type IV collagen exhibit specific distributionpattern. ${ }^{21}$ Nagatsuka et al. studied the expression of anti- $\alpha$ (IV) chain-specific monoclonal antibodies in ameloblastomas, adenomatoid odontogenic tumors, ameloblastic fibromas, ameloblastic carcinomas, ameloblastic fibro-, and primary intraosseous carcinoma. They observed a difference between basement membranes of odontogenic neoplasms, normal oral mucosa and developing tooth germ. Amongst ameloblastomas, desmoplastic showed higher expression of $\alpha 1$ (IV)/ $\alpha 2$ (IV) and $\alpha 5$ (IV)/ $\alpha 6$ (IV) chains than ordinary ameloblastomas. Expression was also observed in basement membrane and tumor cells of AF and ameloblastic fibroodontosarcoma except hard tissues. In AOT, the basement membrane of cribriform areas and hyaline materials were positive, whereas weak around epithelial whorls/rose ttes/nests and mineralized foci. ${ }^{22}$ Malignant odontogenic tumors exhibited irregular $\alpha(\mathrm{IV})$ chain profile as compared to benign odontogenic neoplasms. They exhibited higher $\alpha 5$ (IV)/ $\alpha 6$ (IV) chains compared to $\alpha 1$ (IV)/ $\alpha 2$ (IV) chains. They concluded that $\alpha 5$ (IV)/ $\alpha 6$ (IV) chain deposition play a role in protection by offering resistance to invasion in malignant neoplasms. ${ }^{22}$

\section{Nestin}

Nestin is an intermediate filament. It is related to neurofilaments. It is expressed in muscles, developing a nervous system, bell stage of odontogenesis, functional odontoblasts. Thus, it is related to odontogenesis and dentinal repair. ${ }^{23}$ Fujita et al. (2006) studied nestin expression in ameloblastoma, odontoma, ameloblastic fibroma, ameloblastic fibro-odontoma and fibro-dentinoma, adenomatoid odontogenic tumor, odontogenic fibroma, intra-osseous myxoma. They observed negative nestin expression in ameloblastomas. Odontogenicectomesenchyme adjacent to odontogenic epithelium in mixed odontogenic tumors exhibited positive nestin expression. In odontomas and ameloblastic fibro-odontomas, nestin expressed was observed in odontoblasts and their processes, dentinoid matrix as well as plump cells adhering to dentinoid. Half cases of myxomas and single case of odontogenic fibroma expressed nestin. In AOT, nestin expression was observed in scattered nodular, rosette arrangements as well as in whorled epithelial cells. Thus, they concluded that nestin expression is increased in ectomesenchyme of mixed odontogenic tumors on stimulation by odontogenic epithelium. Also, nesting can be seen as a marker odontoblasts in mixed odontogenic tumors.$^{24}$

\section{Bcl-2 Family Proteins in Odontogenic Tumors}

Apoptosis, also known as programmed cell death, is an essential physiological process for elimination of cells. The protein family involved in apoptosis is Bcl-2. It consists of pro-apoptotic and anti-apoptotic members. Bcl-2 gene homologue, $\mathrm{Bcl}-\mathrm{x}$ gene, encodes two proteins, bcl-xL and bcl-xS. Bcl-xS promotes apoptosis by inhibiting bcl-2 whereas bcl-xL has anti-apoptotic activity. ${ }^{25}$ An additional bcl-2 homolog, bax protein promotes apoptosis by interacting with bcl-2 or bcl-xL proteins. Kumamoto et al. studied the expression of bcl-2, bcl-x and bax proteins in tooth germs and benign and malignant ameloblastomas. They observed intense expression of bcl-2 and bcl-x proteins in peripheral columnar cells of ameloblastomas and weak in central cells. On the other hand, the expression for Bax protein was low. Thus, the proliferative activity of ameloblastomas might be attributed to the antiapoptotic activity of the bcl-2 protein ${ }^{26}$. Bast, Pogrel, andRegezi studied the expression of apoptosis-regulating proteins Bcl-2, Bcl-XL, Bak, and Bax, in OM. An increase in cells staining for anti-apoptotic proteins Bcl-2 and Bcl-X was observed. Pro-apoptotic proteins Bak and Bax were negative. They concluded that OM's grow predominantly because of MMP's and anti-apoptoticproteins like Bcl-2 and Bcl-X. ${ }^{19}$

\section{Calretinin}

Calretinin is a member of a calcium binding protein family. Its molecular weight is $29 \mathrm{kDa}$. Expression of calretinin is observed in certain subtypes of neurons in the central and peripheral nervous systems. Though the exact 
biological role of calretinin is unknown, it is regarded as calciumbuffer or calcium sensor and regulator of apoptosis. Immunohistochemical expression of calretinin was studied by Alaeddini et al. in 20 solid ameloblastomas, 5 CEOT's, 10 AOT's, 10 AF's and 10 OM's. Immunoreactivity was negative in all odontogenic tumors except ameloblastomas. All 20 ameloblastomas showed intense immunopositivity in the nucleus as well as cytoplasm. Expression was seen in stellate reticulum like cells, areas of squamous metaplasia, cells that lined macro and micro-cysts. On the other hand, negative expression was observed in ameloblast like peripheral cells. They concluded that calretinin may have a role in the conversion of remnants of d ental lamina into ameloblastoma. ${ }^{27}$

\section{Syndecan}

Also known as CD138, Syndecan is type-I transmembrane heparan sulfate proteoglycan. Extracellularly, it binds the growth factor and matrix molecules and intracellularly associated with the actin-containing cytoskeleton. Al-Otaibi et al. investigated syndecan expression in 32 ameloblastomas, 26 keratocystic odontogenic tumors (KCOT), and 21 dentigerous cysts. They observed syndecan-1 expression in epithelial and stromal elements. Higher expression was seen in stellate reticulum like cells than ameloblasts. The cystic lining of dentigerous cyst and keratocystic odontogenic tumourshowed higher expression. They observed that mean rank scores of ameloblastoma was lower than these two cystic lesions.They concluded that stromal expression of syndecan-1 is associated with poor prognosis. ${ }^{28}$

\section{Molecular Profiling of Odontogenic Tumors}

Molecular profiling is measuring the expression of multiple genes on tissue samples. Gültekin et al. observed molecular profiling of two AOTs, two ameloblastomas, one $\mathrm{AF}$ and one squamous odontogenic tumor. All tumors showed BRAF (V600E) or KRAS mutations. BRAF mutations were observed in two ameloblastomas and AF. MAPK cascade (Mitogen activated protein kinase) induce and promote cell proliferation. BRAF activates MAPK. Similarly, AOT showed KRAS mutation. Oncogene KRAS belongs to RAS protein family and activates BRAF. ${ }^{29}$

\section{CONCLUSION}

Only a few odontogenic tumor markers are available. Tumor markers will play a significant role even though histopathology seems to be indispensable in the diagnosis of odontogenic tumors.

\section{REFERENCES}

1. Tumor Markers. National Cancer Institute (U S National Institutes of Health [ 2006 Availablefrom: URL:www.cancer. gov.

2. Makretsov NA, Hayes M, Carter BA, Dabiri S, Gilks CB, Huntsman DG. Stromal CD10 expression in invasive breast carcinoma correlates with poor prognosis, estrogen receptor negativity, and high grade. Mod Pathol 2007; 20:84-89.

3. Moradian-Oldak J, Du C, Falini G. On the formation of amelogenin microribbons. Eur J Oral Sci 2006; 114 Suppl 1:289-296.

4. Deutsch D, Haze-Filderman A, Blumenfeld A, Dafni L, Leiser $\mathrm{Y}$, Shay B, et al. Amelogenin, a major structural protein in mineralizing enamel, isalso expressed in soft tissues: Brain and cells of the hematopoietic system. Eur J Oral Sci 2006; 114Suppl 1:183-189.

5. Anigol, et al.: Amelogenin in odontogenic Cysts and tumours: An immunohistochemical study. National Journal of Maxillofacial Surgery 2014;5(2):172.

6. Gao Z et al . Patterns of keratin-expression in rests of Malassez and periapical lesions. J Oral Pathol 1988; 17: 178-185.

7. MM Crivelini, VC de Arau' jo, SOM de Sousa, NS de Arau' jo. Cytokeratins in epithelia of odontogenic neoplasms. Oral Diseases 2003;9:1-6.

8. Chiquet-Ehrismann R, Mackie EJ, Pearson CA, Sakakura T. Tenascin: an extracellular matrix protein involved in tissue interaction during fetal development and oncogenesis. Cell.1986 Oct 10;47(1):131-139.

9. Chiquet-Ehrismann R, Mackie EJ, Pearson CA, Sakakura T. Tenascin: an extracellular matrix protein involved in tissue interaction during fetal development and oncogenesis. Cell 1986;47:131-139.

10. Heikinheimo K, Morgan PR, Happonen RP, Stenmann G, Virtanen I. Distribution of extracellular matrix proteins in odontogenic tumours and developing teeth. VirchowsArchiv B Cell Path01 199;61:101-109.

11. Thesleff I, Mackie E, Vainio S, Ehrismann RC. Changes in the distributionof tenascin during tooth development. Development 1987;101:289-296.

12. Rosen V Cox K. Hatterslev G. Bone morphogenetic protein. In Bilezikian JP, RA!SZ LG, Rodan Ga, eds. Principles of bone biology. San Diego. California: Academic Press. 1996: 661-672.

13. Vainio S, Karavanova I, Jowett A et al. Identification of BMP-4 as a signal mediating secondary induction between epithelial and mesenchymal tissues during early tooth development. Cell1993;75: 45-58.

14. Gao YH, Yang LJ, Yamaguchi A;Imtnunohistochemical demonstration of bone Morphogenetic protein m odontogenictumors. J Oral Pathol Med 1997;26;273-277.

15. Lemer UH. New molecules in the tumour necrosis factor ligand and receptor superfamilies with importance for physiologic and pathological boneresorption. Crit Rev Oral Biol Med 2004;15:64-81.

16. Andrade FR, Sousa DP, Mendonca EF, Silva TA, Lara VS, Batista AC. Expression of bone resorption regulators (RANK,RANKL, and OPG) in odontogenictumors. Oral Surg Oral Med Oral Pathol Oral RadiolEndod 2008; 106(4):548-55.

17. Boyce BF, Xing L. The RANKL/RANK/OPG pathway. CurrOsteoporos Rep.2007 Sep; 5(3):98-104.

18. Sorsa T, Tjaderhane L, Salo T. Matrix metalloproteinases (MMPs) in oral diseases. Oral Dis.2004 Nov;10(6):311-318. 
19. Brian T. Bast, M. Anthony Pogrel, Joseph A. Regezi, The Expression of Apoptotic Proteins and Matrix Metalloproteinases in OdontogenicMyxomas. J Oral MaxillofacSurg2003;61:1463-1466.

20. Pinheiro JJV, Freitas VM, Moretti AIS, Jorge AG, Jaeger RG. Local invasiveness of ameloblastoma. Role played by matrix metalloproteinases and proliferative activity. Histopathology [Internet]. Wiley; 2004 Jul;45(1):65-72.

21. Hudson BG, Reeders ST, Tryggvason K. Type IV collagen: structure, gene organization, and role in human diseases. Molecular basis of Goodpasture and Alport syndromes and diffuse leiomyomatosis. J BiolChem1993 Dec 15;268(35) 26033-26036.

22. Nagatsuka H, Siar C, Nakano K, Tsujigiwa H, Gunduz M, Choufuku H, et al. Differential expression of collagen IV 1 to 6 chains in basement membranes of benign and malignant odontogenic tumors. Virchows Archiv [Internet]. Springer Nature; 2002 Oct 1;441(4):392-399

23. About I, Laurent-Maquin D, Lendahl U, Mitsiadis TA. Nestin Expression in Embryonic and Adult Human Teeth under Normal and Pathological Conditions. The American Journal of Pathology [Internet]. Elsevier BV; 2000 Jul;157(1):287-295.
24. Fujita S. Nestin expression in odontoblasts and odontogenic ectomesenchymal tissue of odontogenic tumours. Journal of Clinical Pathology BMJ; 2006 Mar 1;59(3):240-245.

25. Tsujimoto Y. Role of Bcl-2 family proteins in apoptosis: apoptosomes or mitochondria? Genes to Cells. 1998 Nov;3(11):697707.

26. Kumamoto H, Ooya K. Immunohistochemical analysis of bcl-2 family proteins in benign and malignant ameloblastomas. Journal of Oral Pathology \& Medicine. 2007 Feb 27;28(8):343-349.

27. Alaeddini M, Etemad-Moghadam S, Baghaii F. Comparative expression of calretinin in selected odontogenic tumours: a possible relationship to histogenesis. Histopathology 2008 Feb;52(3):299-304.

28. Al-Otaibi O, Khounganian R, AnilS, Rajendran R. Syndecan-1 (CD 138) surface expression marks cell type and differentiation in ameloblastoma, keratocystic odontogenic tumor, and dentigerous cyst. Journal of Oral Pathology \& Medicine. 2012 Jul 2;42(2):186-193.

29. Gültekin SE, Sengüven B, Aziz R, Heydt C, Buettner R. Molecular Profiling of Odontogenic Tumors - Pilot Study. Balkan Journal of Dental Medicine. 2017 Jul 26;21(2):112-115. 